\title{
ON THE CONJUGACY PROBLEM AND GREENDLINGER'S EIGHTH-GROUPS
}

SEYMOUR LIPSCHUTZ

1. Introduction. In $1912 \mathrm{Max}$ Dehn [1], [2] solved the conjugacy problem for the fundamental group $G_{k}$ of a closed 2-manifold of genus $k$; this group can be finitely presented as follows:

$$
G_{k}=g p\left(a_{1}, b_{1}, \cdots, a_{k}, b_{k} ; a_{1}^{-1} b_{1}^{-1} a_{1} b_{1} \cdots a_{k}^{-1} b_{k}^{-1} a_{k} b_{k}=1\right) .
$$

In 1960 Greendlinger [3] solved the conjugacy problem for the class of eighth-groups; we note that this class contains the above groups $G_{k}$ for $k \geqq 3$. Further generalizations of Dehn's work on the conjugacy problem were obtained again by Greendlinger [4] and also by Schupp [11] using methods developed by R. C. Lyndon [9].

In this paper, using a theorem of Solitar [10, Theorem 4.6], we solve the conjugacy problem for certain generalized free products of eighth-groups; namely,

MAIN THEOREM. Let $G$ be the free product of eighth-groups $A_{\lambda}$ with a cyclic group $H$ amalgamated where $H$ is generated by a basic element $h$ in each factor. Then $G$ has a solvable conjugacy problem.

We remark that the relevant notation and definitions appear either in $\$ 2$ or in Magnus, Karrass and Solitar [10]. Our main technical result Lemma 3 uses a result of Greendlinger [3, Theorem V] which we restate below.

2. Notation and definitions. Let $A$ be a group generated by $a_{1}, \cdots, a_{\mu}$ with defining relations $R_{1}=1, \cdots, R_{\gamma}=1$. We denote this group by

$$
A=g p\left(a_{1}, \cdots, a_{\mu} ; R_{1}=1, \cdots, R_{\gamma}=1\right),
$$

where we call the $R_{i}$ relators. We will assume (without loss in generality) that the relators $R_{i}$ have been symmetrized, that is, that the $R_{i}$ are cyclically reduced and include inverses and cyclic transforms of each other. We call $A$ an eighth-group if, for any distinct relators $R_{1} \equiv X Y$ and $R_{2} \equiv X Z$, the length of the common initial segment $X$ is less than $1 / 8$ of the length of either relator.

We use the following notation for words $u$ and $v$ in the group $A$ :

Received by the editors February 27, 1969. 
$l(u)$ for the length of $u$,

$u=v$ means $u$ and $v$ are the same element of the group $A$,

$u \approx v$ means $u$ and $v$ are freely equal,

$u \equiv v$ means $u$ is identical to the word $v$,

$u \wedge v$ means $u$ does not react with $v$, i.e. $w \equiv u v$ is freely reduced,

$u \subset v$ means $u$ is a subword of $v$, i.e. $v \equiv w_{1} u w_{2}$.

We say $v$ cyclically contains $u$ if a cyclic transform of $v$ contains $u$ as a subword.

We introduce the notation

$$
u<p / q R
$$

which means that $u$ does not contain $p / q$ or more of any defining relator $R_{i}$, that is, if $u$ and $R_{i}$ contain the same subword $S$ then $l(S)$ $<p / q l\left(R_{i}\right)$. We define $u \leqq p / q R$ analogously. We assume obvious properties of this definition such as: (i) if $u<1 / 8 R$ and $v<1 / 8 R$ then $u v<2 / 8 R$, (ii) if $u \subset v$ and $v<1 / 8 R$ then $u<1 / 8 R$, (iii) if $T<1 / 8 R$ and $T \subset R_{i}$ then $l(T)<1 / 8 l\left(R_{i}\right)$. We also assume, unless otherwise stated or implied, that all elements are written as freely reduced words $w \leqq 4 / 8 R$.

Definition. An element $h$ in an eighth-group $A$ is said to be basic if $h$ is cyclically reduced, has infinite order, and is $<1 / 8 R$.

The condition $h<1 / 8 R$ implies, by Lemma 2 below, that $h^{m}<2 / 8$ $R$ for any integer $m$. (We note [3, Theorem VIII] that $u$ in $A$ has finite order only if there is a relator $R^{\prime} \equiv S^{n}$ where $u$ is a conjugate of a power of $S$.)

3. Theorems. Let $u$ and $v$ be cyclically reduced elements in the group $G$ of the Main Theorem, and suppose $u$ and $v$ are conjugate in $G$. By Solitar's theorem [10, Theorem 4.6] $u$ and $v$ have the same length $n$. The following theorem gives additional conditions on $u$ and $v$; here $r$ denotes the length of the largest defining relator of those factors $A_{\lambda}$ to which $u$ or $v$ belong.

Theorem 1. If $n=1$, say $u \in A_{i}$ and $v \in A_{j}$, then either $u$ and $v$ are conjugate in $A_{i}=A_{j}$ or else $u$ and $v$ are conjugate to $h^{t}$ in $A_{i}$ and $A_{j}$ respectively where

$$
|t| \leqq r^{2}+r l(u) .
$$

If $n>1$, then $h^{m} u^{*} h^{-m}=v^{*}$ where $u^{*}$ and $v^{*}$ are cyclic transforms of $u$ and $v$ respectively and

$$
|m| \leqq l(u)+l(v)+2+r .
$$


Proof. Suppose $n=1$ and $u$ and $v$ are not conjugate in a factor. By Solitar's theorem there is a sequence of elements $u, h_{1}, h_{2}, \cdots$, $h_{s}, v$ where the $h_{i}$ belong to the amalgamated subgroup $H$ and consecutive terms of the sequence are conjugate in a factor. By Theorem 2 of [5], powers of $h$ are in different conjugate classes in each factor; hence $h_{1}=\cdots=h_{s}=h^{t}$. Thus $u$ and $v$ are conjugate to $h^{t}$ in $A_{i}$ and $A_{j}$ respectively. Furthermore, by the Theorem of [7], we have $|t| \leqq r^{2}+r l(u)$, as required.

Now suppose $n>1$. By Solitar's theorem, there exist cyclic transforms $u^{*}$ and $v^{*}$ of $u$ and $v$ respectively which are conjugate in $G$ by an element in the amalgamated subgroup $H$, i.e. $h^{m} u^{*} h^{-m}=v^{*}$ for some integer $m$. It remains to show that $m$ satisfies the condition stated in the theorem. Suppose $u^{*}$ and $v^{*}$ have normal forms $u^{*}$ $=x_{1} x_{2} \cdots x_{n}$ and $v^{*}=y_{1} y_{2} \cdots y_{n}$. Then

$$
h^{m} x_{1} x_{2} \cdots x_{n} h^{-m}=y_{1} y_{2} \cdots y_{n} .
$$

If $h$ commutes with each of the $x$ 's then we can choose $m=0$. On the other hand, let $k$ be the smallest in teger such that $h$ does not commute with $x_{k}$. Then $y_{k}^{-1} h^{m} x_{k}$ belongs to the amalgamated subgroup $H$; say $y_{k}^{-1} h^{m} x_{k}=h^{-s}$. Then $h^{m} x_{k} h^{s}=y_{k}$. By Lemma 4 below,

$$
|m| \leqq l\left(x_{k}\right)+l\left(y_{k}\right)+2+r \leqq l(u)+l(v)+2+r .
$$

Thus Theorem 1 is proved.

Proof of Main Theorem. By the above theorem, the conjugacy problem in $G$ is reduced to a finite number of conjugacy problems in factors of $G$ or to a finite number of word problems in $G$. The Main Theorem now follows from the fact that the conjugacy problem has been solved for the factors by Greendlinger [3] and the word problem has been solved for $G$ by Lipschutz [6].

4. Lemmas. We first state Greendlinger's basic result on eighthgroups [3, Theorem V] which we need for our main technical result Lemma 3.

GreENDLINGER'S Lemma. Let $W$ be a cyclically reduced nonempty word in an eighth-group $A$ and suppose $W=1$. Then either $W$ is a defining relator or else $W$ cyclically contains disjointly

(i) $>7 / 8$ each of two $R$ 's,

(ii) $>6 / 8$ each of three $R$ 's,

(iii) $>6 / 8$ each of two $R$ 's and $>5 / 8$ each of two $R$ 's, or

(iv) $>5 / 8$ each of five $R$ 's.

Lemma 1. Suppose in an eighth-group $A$ there is a defining relator 
$R_{1} \equiv W^{n} Y T$ where $n>1$ and $Y$ is an initial segment of $W$, i.e. $W \equiv Y X$. Then either $W$ has finite order, or else $W^{n-1} Y<1 / 8 R$ and $W^{n} Y<2 / 8 R$.

Proof. Consider the defining relator $R_{2} \equiv W^{n-1} Y T W$. Note $W^{n-1} Y$ is a common initial segment of $R_{1}$ and $R_{2}$. Suppose $R_{1} \not \equiv R_{2}$. Then by definition of an eighth-group $W^{n-1} Y<1 / 8 R$. Now $W \subset W^{n-1} Y$ because $n>1$. Hence $W<1 / 8 R$ and therefore $W^{n} Y<2 / 8 R$. On the other hand, suppose $R_{1} \equiv R_{2}$. Then $W$ and $W^{n-1} Y T$ commute as words in a free group and hence are each freely equal to powers of the same word $U$. Accordingly, $R_{1}$ is a power of $U$ and hence $U$ has finite order in the eighth-group $A$. But $W$ is a power of $U$; hence $W$ also has finite order in $A$.

Lemмa 2. Let $u$ be a cyclically reduced element of infinite order in an eighth-group $A$. If $u<1 / 8 R$, then $u^{m}<2 / 8 R$ for any integer $m$.

Proof. Suppose $S$ is a subword of $u^{m}$ and some defining relator $R$. Then $S \equiv(Y X)^{n} Z$ where $u \equiv X Y$ and $Y X \equiv Z W$. We need to show that $l(S)<2 / 8 l(R)$ or equivalently $S<2 / 8 R$.

Case I. $n=0$; that is, $S \equiv Z$. Now $Y, X \subset u$ and $u<1 / 8 R$; hence $S \equiv Z \subset Y X$ and so $S<2 / 8 R$.

Case II. $n>1$. By Lemma $1, S<2 / 8 R$.

Case III. $n=1$; that is, $S \equiv Y X Z$. Suppose $Z$ is an initial segment of $Y$. Then $X Z \subset X Y \equiv u$; hence $X Z<1 / 8 R$, and $Y<1 / 8 R$. Accordingly, $S \equiv Y X Z<2 / 8 R$. On the other hand, suppose $Y$ is an initial segment of $Z$; say $Z \equiv Y U$. Then $S \equiv Y X Z \equiv Y X Y U$ where $U$ is a subword of $X$. We have

$l(u)=l(X Y)<1 / 8 l(R)$ and $l(Y)+l(U) \leqq l(X Y)<1 / 8 l(R)$.

Therefore $l(S)<2 / 8 l(R)$.

Thus the lemma is proved.

Lemma 3. Let $W \equiv P X Q Y$ be a cyclically freely reduced nonempty word in an eighth-group $A$ with $P, Q<2 / 8 R$ and $X, Y \leqq 4 / 8 R$. If $W=1$, then $X$ and $Y$ are nonempty and $l(P) \leqq \max l\left(R_{i}\right)$.

Proof. We write $W$ around a square as in Figure 1 . Since $P, Q$ $<2 / 8 R$, if $W$ cyclically contains $>6 / 8$ of a defining relator then it must cross at least two corners of the square, and if $W$ cyclically contains $>5 / 8$ of a defining relator then it must cross at least one corner of the square. Accordingly, $W$ cannot satisfy conditions (ii), (iii) or (iv) of Greendlinger's lemma. Furthermore, if $X$ is empty then: (1) $W \equiv P Q Y<8 / 8 R$ and cannot be a defining relator, and (2) $W$ cannot satisfy condition (i) of Greendlinger's lemma. Accordingly, $X$ and similarly $Y$ are nonempty words. 


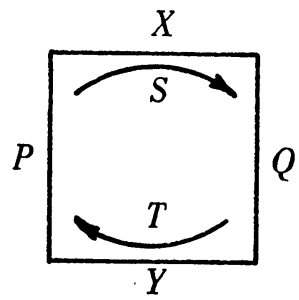

It remains to show that $l(P) \leqq \max l\left(R_{i}\right)$. If $W$ is a defining relator then obviously $l(P) \leqq \max l\left(R_{i}\right)$. On the other hand, suppose $W$ satisfies condition (i) of Greendlinger's lemma. Specifically, suppose $W$ cyclically contains disjointly $S$ and $T$ where there are defining relators $R_{1} \equiv S G^{-1}$ and $R_{2} \equiv T H^{-1}$ with $l(S)>7 / 8 \quad l\left(R_{1}\right)$ and $l(T)$ $>7 / 8 l\left(R_{2}\right)$. We also assume that $S$ and $T$ are as large as possible. Except for symmetry, there are only two ways that $S$ and $T$ can lie in a cyclic transform of $W$ :

Case I. $P \subset S$ and $Q \subset T$. Then $l(P) \leqq l(S) \leqq l\left(R_{1}\right) \leqq \max l\left(R_{i}\right)$.

Case II. $X \subset S$ and $Y \subset T$, as indicated in Figure 1. Say $P \equiv E_{2} E E_{1}$, $Q \equiv F_{1} F F_{2}, S \equiv E_{1} X F_{1}$ and $T \equiv F_{2} Y E_{2}$. Replacing $S$ by $G$ and $T$ by $H$ in a cyclic transform of $W$, we obtain $V \equiv E G F H=1$. We note that the maximality of $S$ implies $E \wedge G$. (Otherwise, if $E \equiv E^{\prime} a$ and $G \equiv a^{-1} G^{\prime}$ then we could have chosen $S^{\prime} \equiv a S$ in place of $S$.) Similarly, $G \wedge F, F \wedge H$ and $H \wedge E$. However, applying Greendlinger's lemma to $V$, we see that $V$ must freely reduce to the empty word because $E$, $F<2 / 8 R$ and $G, H<1 / 8 R$. Accordingly, either $E, F, G$ or $H$ must be empty. But $P X Q, Q Y P<8 / 8 R$; hence $S, T<8 / 8 R$ and therefore $G$ and $H$ are not empty words. Thus $E$ or $F$ is empty. Now $P<2 / 8 R$ implies $l\left(E_{1}\right)<2 / 8 l\left(R_{1}\right)$ and $l\left(E_{2}\right)<2 / 8 l\left(R_{2}\right)$. Consequently, if $E \equiv 1$ then $P \equiv E_{2} E_{1}$ and

$$
l(P)=l\left(E_{1}\right)+l\left(E_{2}\right)<2 / 8\left[l\left(R_{1}\right)+l\left(R_{2}\right)\right]<\max l\left(R_{i}\right) .
$$

On the other hand, suppose $F \equiv 1$. Then $V \equiv E G H$ and, since $V$ freely reduces to the empty word,

$$
l(E) \leqq l(G)+l(H)<1 / 8\left[l\left(R_{1}\right)+l\left(R_{2}\right)\right] .
$$

Furthermore, since $P \equiv E_{2} E E_{1}$,

$$
l(P)=l(E)+l\left(E_{1}\right)+l\left(E_{2}\right)<3 / 8\left[l\left(R_{1}\right)+l\left(R_{2}\right)\right]<\max l\left(R_{\mathbf{i}}\right) .
$$

Thus the lemma is proved.

Lemma 4. Let h be a basic element in an eighth-group $A$, and suppose $h$ does not commute with an element $x \in A$. If $W \equiv h^{m} x h^{s} y=1$ then 


$$
|m| \leqq l(x)+l(y)+2+\max l\left(R_{i}\right) .
$$

Proof. We need only consider the case $m>l(x)+2$. If $W$ is freely equal to 1 , then by Corollary 1 in [8] we have $|m| \leqq l(x)+l(y)+2$.

On the other hand, suppose $W \neq 1$, and say $W$ freely cyclically reduces to $V \equiv P X Q Y$ where $P \subset h^{m}, X \subset x, Q \subset h^{s}$ and $Y \subset y$. By Lemma $2, h^{m}, h^{s}<2 / 8 R$; hence $P, Q<2 / 8 R$. Also, $X, Y \leqq 4 / 8 R$. By Lemma 3, $X$ and $Y$ are nonempty words. Accordingly,

$$
l(P) \geqq l\left(h^{m}\right)-l(x)-l(y) .
$$

But by Lemma $3, l(P) \leqq \max l\left(R_{i}\right)$; hence

$$
|m| \leqq l\left(h^{m}\right) \leqq l(P)+l(x)+l(y) \leqq l(x)+l(y)+\max l\left(R_{i}\right) .
$$

Thus the lemma is proved.

\section{BIBLIOGRAPHY}

1. M. Dehn, Über unendliche diskontinuierliche Gruppen, Math. Ann. 71 (1911), $116-144$

2. - Transformation der Kurven auf zweiseitigen Flachen, Math. Ann. 72 (1912), 413-421.

3. M. Greendlinger, On Dehn's algorithm for the conjugacy and word problems, with applications, Comm. Pure Appl. Math. 13 (1960), 641-677.

4. - On the word problem and the conjugacy problem, Izv. Akad. Nauk SSSR Ser. Mat. 29 (1965), 245-268.

5. S. Lipschutz, On powers of elements in S-groups, Proc. Amer. Math. Soc. 13 (1962), 181-186.

6. - An extension of Greéndlinger's results on the word problem, Proc. Amer. Math. Soc. 15 (1964), 37-43.

7. —_, Powers in eighth-groups, Proc. Amer. Math. Soc. 16 (1965), 1105-1106.

8. - Generalization of Dehn's result on the conjugacy problem, Proc. Amer. Math. Soc. 17 (1966), 759-762.

9. R. C. Lyndon, On Dehn's algorithm, Math. Ann. 166 (1966), 208-228.

10. W. Magnus, A. Karrass and D. Solitar, Combinatorial group theory, Wiley, New York, 1965.

11. P. E. Schupp, On Dehn's algorithm and the conjugacy problem, Math. Ann. 178 (1968), 119-130.

\section{TEMPLE UNIVERSITY}

